\title{
Technology-facilitated Societal Consensus
}

\author{
Timotheus Kampik \\ Department of Computing Science \\ Umeå University \\ Umeå, Sweden \\ tkampik@cs.umu.se
}

\author{
Amro Najjar \\ Department of Computing Science \\ Umeå University \\ Umeå, Sweden \\ AI-Robolab/ICR \\ Computer Science and Communications \\ University of Luxembourg \\ Esch-sur-Alzette, Luxembourg \\ najjar@cs.umu.se
}

\begin{abstract}
The spread of radical opinions, facilitated by homophilic Internet communities (echo chambers), has become a threat to the stability of societies around the globe. The concept of choice architecture-the design of choice information for consumers with the goal of facilitating societally beneficial decisions-provides a promising (although not uncontroversial) general concept to address this problem. The choice architecture approach is reflected in recent proposals advocating for recommender systems that consider the societal impact of their recommendations and not only strive to optimize revenue streams. However, the precise nature of the goal state such systems should work towards remains an open question. In this paper, we suggest that this goal state can be defined by considering target opinion spread in a society on different topics of interest as a multivariate normal distribution; i.e., while there is a diversity of opinions, most people have similar opinions on most topics. We explain why this approach is promising, and list a set of crossdisciplinary research challenges that need to be solved to advance the idea.
\end{abstract}

\section{KEYWORDS}

Choice architecture, Recommender systems, Persuasion

ACM Reference Format:

Timotheus Kampik and Amro Najjar. 2019. Technology-facilitated Societal Consensus. In 27th Conference on User Modeling, Adaptation and Personalization (UMAP '19 Adjunct), fune 9-12, 2019, Larnaca, Cyprus. ACM, New York, NY, USA, 5 pages. https://doi.org/10.1145/3314183.3323451

\section{INTRODUCTION}

The technology-facilitated erosion of societal consensus has been a topic of intense discussion in and beyond the academic community since the publication of Sunstein's Rebublic.com [45] and gained further attention after the events around the 2016 United States presidential election, the rise of far-right parties in Europe [1, 17], and the vote for the Brexit [9]. Socio-economic problems (in particular, increasing income inequality [38]) are likely facilitating these

This work is licensed under a Creative Commons Attribution-NonCommercial International4.0 License.

UMAP'19 Adjunct, June 9-12, 2019, Larnaca, Cyprus

(C) 2019 Copyright held by the owner/author(s).

ACM ISBN 978-1-4503-6711-0/19/06.

https://doi.org/10.1145/3314183.3323451 developments. In the wake of these events, activists call for the development of (social) technology that prioritizes the wellbeing of individual users and society as a whole over monetary rewards for application designers, investors, or other potentially manipulative stakeholders [36]. In this paper, we suggest that the strength of opinions on a set of relevant topics can be modeled as a multivariate distribution and that the shape of the distribution can be used as an indicator of the health of societal opinion discourse. We propose that an interdisciplinary, data-driven approach can be used to develop information systems that employ this indicator to facilitate social consensus and rational discourse.

\section{BACKGROUND}

To motivate our work and ground it in the state-of-the-art, we provide an overview of emerging research on technology-facilitated social tensions, as well as of recent developments around the concept of user-centered technology.

\subsection{Technology-facilitated Social Tensions}

Both in popular and scientific literature, the effect of technologyfacilitated social influence phenomena on society is a topic of intense interest and debate. Below, we describe and critically examine the most prominent phenomena:

Filter bubbles. According to Eli Pariser, who coined the term in popular literature, filter bubbles emerge when (social) Internet applications extrapolate data on a user's preferences, social contacts, and interests to select what content to show to this user [35]. Hence, filter bubbles create a unique universe of information for each user. It is not clear to what extent filter bubbles are de facto a prevalent Internet phenomenon; while empirical studies exist, they are typically limited in scope and find no [16,30] to some [3] evidence.

Echo chambers. The term echo chamber refers to "a bounded, enclosed media space that has the potential to both magnify the messages delivered within it and insulate them from rebuttal" [18]. When users are in an echo chamber, their beliefs are likely to be reinforced and their positions radicalized because new information they receive is typically aligned with their pre-existing beliefs. Empirical evidence for the existence of echo chambers exists (e.g. [2]), although the strength of the phenomenon is questioned by some researchers [34]. 
Information cascades. Information cascades occur when it appears optimal for an individual, having observed the actions of those acting ahead of them, to follow the others' behavior without regarding their own information [5]. Consequentially, the social equilibrium may suffer a radical shift; masses of individuals converge to one action, or one ideological position, on the basis of little information. Several approaches have been proposed to model information cascades. These approaches are either grounded in opinion dynamics (e.g. $[15,20])$ or game theory (e.g. $[24,31])$. In the first family of models (e.g. [15, 20,48]), the aim of agents in the network is to spread their information, whereas in the second type (e.g. [24, 31]) agents seek to maximize the payoff of their utility function. Recent studies provide evidence for information cascades on social media (e.g., Twitter) [39] and person-toperson recommender systems for online retail [25].

Fake news. Fake news can be defined as fabricated information that mimics media content in form but not in organizational processes or intent [23]. Fake news are spread by outlets lacking the editorial norms that were established in the first half of the 20th century to ensure accuracy and credibility of information. Despite being a technology-agnostic concept, the spread and influence of fake news witnessed a significant increase with the advent of online social networks, which have become one of the main sources of information for large parts of the population [43]. The impact of fake news conveyed on social network platforms is aggravated by two factors: (i) social: aside from reaching the user as integrated and openly available content provided through social network platforms, fake news can benefit from the network of the users' relatives and friends (i.e., a piece of fake news shared by close friends can have a stronger impact on the receiver). Furthermore, the emergence of homogeneous social networks accentuated the problems of homophilia, which increases polarization and reduces tolerance for alternative views. (ii) technical: the use of social bots [13], whose number is estimated to be in the order of tens of millions on Facebook and Twitter [47], can facilitate the spread of fake news on these social networks and allow fake news and propaganda publishers to automatize and scale their fake news production and distribution [42]. The activity of social bots intensifies during critical political campaigns, e.g., during the presidential elections in the USA [37] and France [12], and during the 2017 German federal elections [32].

One can conclude that-although the impact strength of some of the aforementioned phenomena is disputable-evidence exists for a) increased erosion of the political center in societies across the globe and $b$ ) a link between this erosion and the aforementioned social media phenomena.

\subsection{Behavioral Economics and User-centered Technology}

The field of behavioral economics emerged in the second half of the 20th century and revolutionized economic research and decision making by acknowledging that human agents in markets are typically not acting rationally [19]. Thaler et al. have built upon behavioral economics to devise the concept of choice architecture [4], which describes the preparation of information in a way that helps agents of limited rationality make good decisions. In addition, Thaler et al. have devised the related maxim of libertarian parternalism [46], which advocates a benevolent, limitedly paternalistic state that allows its citizens to make decisions freely (libertarian) but arranges choice information in a way that facilitates decision-making in the interest of society (paternalistic). Recently, the initial works on choice architecture and libertarian paternalism have been criticized for primarily approaching the issue from a utopian perspective and not thoroughly addressing manipulative aspects of choice architecture [34]. Indeed, data analytics firm Cambridge Analytica, who played a major role in a social media data privacy scandal, has reportedly employed choice architecture concepts for purely manipulative purposes [50].

The issues outlined by the critics of choice architecture are reflected in socio-technical problems with personalized information technology. Such technology typically makes use of recommender systems, which suggest content or items for users based on the preferences of their friends (collaborative filtering), on their demographic data (e.g. age), or on previous user behavior for personalization purposes [6]. The potentially damaging social effects of recommender systems are well described in academic literature. Vulnerability against manipulation is one of the key concerns. Manipulation occurs when some users provide dishonest preferences to skew the recommendation (also called shilling) [21]. Further research suggests that even simple attack techniques can significantly distort the recommendations provided by common recommender algorithms [22, 29]. Recent research establishes the need to explore how recommender systems can be designed and configured to achieve positive social outcomes and to "put [...] the user in control" [21]. The aforementioned developments are well-known by industry practitioners. In particular, the Center for Humane Technology, founded by a number of early employees of Silicon Valley tech giants, spearheads the so-called time well spent-movement of industry practitioners who argue that consumer information technology is too often designed to maximize the time the user spends with an application or device and want to shift the focus back to optimizing utility for the user [36].

\section{AN OPINION DISTRIBUTION HEALTH MEASURE THAT INFORMS RECOMMENDER SYSTEM BEHAVIOR}

To address the problem we introduced above, we propose a model that provides a simplified perspective on the state of societal consensus (the opinion distribution among members of society/citizens) on a particular topic of interest as a univariate distribution. Such a topic of interest would typically be a potential or existing policy, for example the legal recognition of same-sex marriages; the opinion strength is a quantification of an individual's support or disapproval. Figure 1 shows three example consensus states on a topic:

- Social tensions (parabolic distribution): society is divided on the topic. Citizens tend to have an extreme opinion, which is equally likely to be on either side of the scale; 




Figure 1: Distributions that indicate social tensions (red), healthy discourse (blue, dashed), and opinion dictate/stagnation (black, dotted); the vertical axis indicates opinion density, the horizontal axis opinion strength for or against a given policy.

- Dictatorship/Stagnation (low-variance Gaussian): although almost all citizens have approximately the same opinion, variance is not tolerated and societal evolution on the topic stalls. This can either be the case if people avoid expressing diverging opinions in fear of retaliation ${ }^{1}$, or if societal consensus on a given topic has been reached.

- Healthy discourse (high-variance Gaussian): citizens tend to have similar opinions on the topics. However, there is still a diversity of opinions that allows for societal progress.

It can be expected that interdependencies between opinions on different topics exist. For example, when an event causes an individual to change their opinion on reproductive rights, it could be that the same event also affects this individual's opinion on parental benefits. Hence, it appears reasonable to model the opinion distribution of all ( $n$ ) relevant opinions as an $n$-dimensional multivariate distribution. The model reflects Nietzsche's concept of moral values as "formations in the mountain of humantiy" [33, p. 63] (although it takes it to new dimensions). Figure 2 presents a simplified bivariate example.

We suggest that social media and other content-serving applications can facilitate societal consensus as follows:

(1) Determine the opinion distribution by mining topics of societal relevance and assessing opinion strength;

(2) Assess the health of the opinion distribution based on the collected opinion strength information.

(3) Derive actions from the health assessment that adjust the behavior of the recommender systems to provide recommendations that are likely to move the opinion strength distribution to a "healthy" shape.

The overall architecture can be viewed as a socio-technical multiagent system (see, e.g. Wooldridge [49]): end-users, different applications (which need to align their behavior to prevent a tug of

\footnotetext{
${ }^{1}$ In this case, the stable opinion might be extreme in comparison to other societies.
}

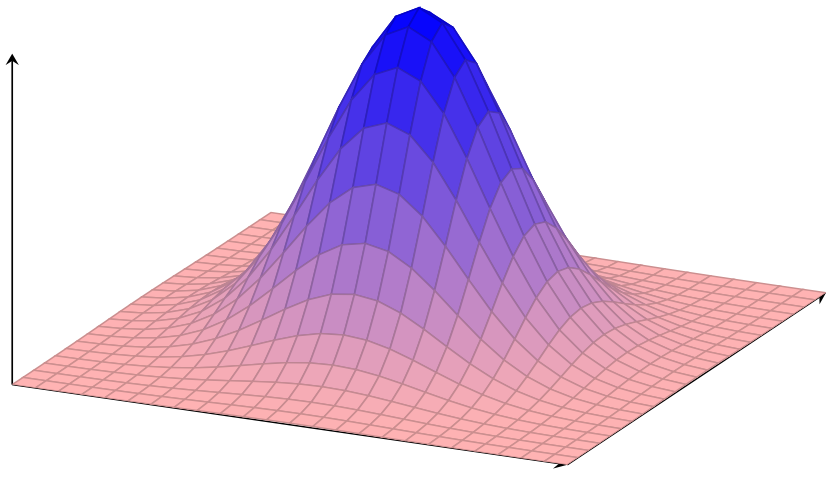

Figure 2: Simplified (bivariate) example of a multivariate target distribution: radical fringes in light red; strong, moderate center in dark blue; the vertical axis indicates opinion density, the horizontal axes indicate opinion strength, e.g. for or against reproductive rights and parental benefits, respectively.

war of opinion influencing), and a regulating body (which imposes rules the applications need to follow) can be seen as agents (see: Figure 3). The proposed approach aligns well with open research problems as identified by scientists from different disciplines. It can be considered a "system enhancer" and "information organizer" as proposed by Sen et al. [41], as well as an application of "smart nudges" as proposed by the psychologists and economists Smith et al. [44].

\section{SOCIO-TECHNICAL CHALLENGES AND INTERDISCIPLINARY SOLUTIONS}

In this section, we explain the three-step process introduced above (determine opinion distribution, assess opinion distribution health, and derive actions). Along the way, we highlight related socio-technical challenges and potential interdisciplinary solutions.

\subsection{Determining the Opinion Distribution}

To determine a multivariate distribution of opinion strengths on all topics of societal importance, one first needs to decide which topics are relevant (topic identification) and then assess the strengths of all available existing opinions on all given topics (sentiment analysis). For topic identification and sentiment analysis, well established tools and methods are available (see, for example: [27]). However, open research challenges exist, as the opinion strength distribution needs to be determined across a complex landscape of diverse information systems, and the positions of potentially "silent" individuals needs to be estimated. Moreover, the health indicator should consider interdependencies between opinions on different topics. For this, methods like word vector-based sentiment analysis [28] can be adjusted and applied. To mine accurate opinions and to provide effective recommendations, an approach similar to the 


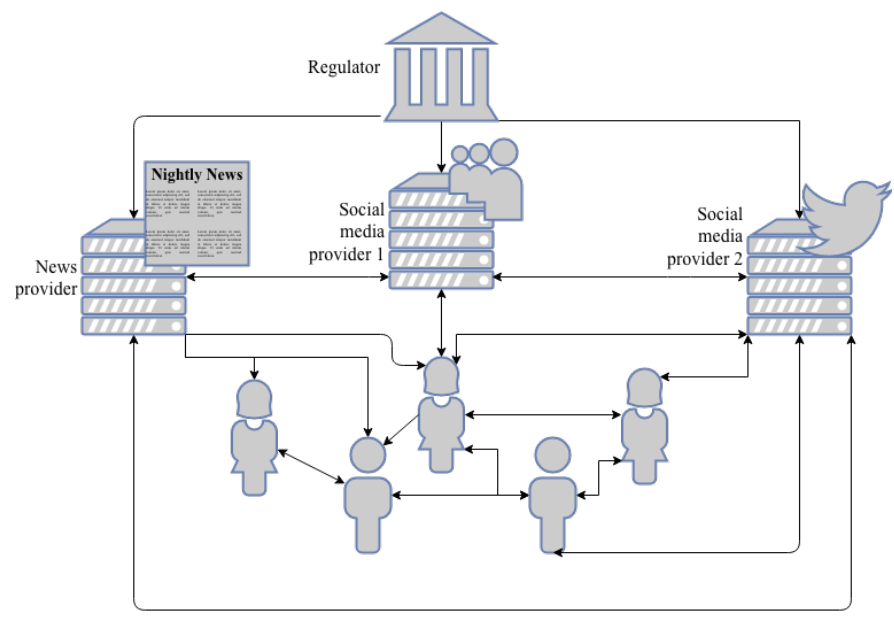

Figure 3: Socio-technical landscape as a multi-agent system system.

holistic recommendation concept as proposed by Cena et al. [7] can be employed.

\subsection{Assessing the Health of the Opinion Distribution}

When the opinion strength distribution has been determined, its health-i.e., the state of societal consensus-needs to be assessed in the best possible way. For this, a simple indicator, similar to the Gini coefficient for measuring income inequality [14] could be devised ${ }^{2}$. In addition to considering the properties of a static snapshot of the opinion strength distribution at a specific point in time (for example: the variance), one should consider the rate of opinion change; i.e., a too fast rate of change could imply instability, whereas a lack of change over time could imply a stalemate in the competition of opinions.

A related challenge is the reinforcement of rational and trustworthy opinions. Treating all opinions equally, no matter how abstruse or well-reasoned they are, can hinder an enlightened evolution of opinions. Hence, we propose that content that facilitates rational and trustworthy opinions should be preferred over content of dubious quality. In the spirit of Leibniz, who stated that if "controversies were to arise, there would be no more need of disputation; [it would suffice to say:] let us calculate" [40, p. 170], formal argumentation (in particular: argument mining [26]) can be applied to assess the rationality of opinions.

\subsection{Deriving Actions (Recommender System Behavior)}

Based on the health assessment of the opinion strength distribution, the behavior of the applications' recommender systems need to be adjusted, with the goal to move from the current opinion strength distribution to a target distribution; i.e., the recommender systems should present content to users that is likely to prevent opinion

\footnotetext{
${ }^{2} \mathrm{~A}$ discussion of the strengths and weaknesses of the Gini coefficient is beyond the scope of this paper.
}

radicalization or expected to facilitate opinion diversification (depending on a given user's opinion and the current opinion strength distribution). To assess the impact of changes in recommender system behavior, social multi-agent system simulations [8] can be employed. Alternatively, one can use control-theoretical approaches as applied by other self-adaptive software like cloud computing environments [11] to implement self-adapting recommender systems. To optimize the impact of individual recommendations, techniques that consider the open-mindedness of a user when deciding on how "diverse" (i.e., how divergent from the user's current opinion) a recommendation should be-as suggested by Eskandanian et al. [10]-can be employed.

\section{SOCIO-ETHICAL CONCERNS}

Considering the social nature of the problem at hand, the proposed approach comes with a set of socio-ethical implications. Like any application of choice architecture (c.f. [34]), our approach raises the question to what extent it is facilitating societal welfare and to what extent it is manipulating users in the interests of the system designers. An important concern is the implementation of governance and abuse prevention mechanisms that ensure the actions the system determines are in fact benefiting society as a whole and not a limited number of influential stakeholders. While the proposed concept is meant to be applied to prevent the erosion of societal consensus and to mitigate societal tensions without stifling discourse, a conceptually similar approach can be employed by authoritarian governments to ensure that opinions do not diverge from the regime's doctrine. A technical solution to prevent this form of misuse appears to be out of reach. Instead, the ethical nature of the application is dependent on the organization that governs it. As outlined by Sunstein [45], incentives for implementing societally beneficial types of choice architecture can either be provided through regulation or consumer choice. In light of the complexity of the socio-technical system in focus, we regard regulation as the more realistic option. Critics of choice architecture might argue that the solution to the problem is not the implementation of recommender systems that try to improve societal consensus, but the abolishment of recommender systems in general. However, such an approach would ignore the positive impact recommender systems can have on user experience (if applied in the interest of the user), as well as the potential of recommender systems to dissolve tensions caused by unrelated phenomena.

The proposed architecture is merely a partial solution to the problem in focus; it is unlikely that the problems at hand can be solved with a technocratic approach alone. Society's ability to engage in an enlightened, democratic discourse that informs public policy is a necessary condition for the approach to work. The proposed concept has the potential to make a society with a healthy discourse culture and working democratic institutions more resilient, but can hardly move a strongly divided or authoritarian society to a state of enlightened societal discourse.

\section{ACKNOWLEDGMENTS}

This work was partially supported by the Wallenberg AI, Autonomous Systems and Software Program (WASP) funded by the Knut and Alice Wallenberg Foundation. 


\section{REFERENCES}

[1] Hunt Allcott and Matthew Gentzkow. 2017. Social media and fake news in the 2016 election. Journal of Economic Perspectives 31, 2 (2017), 211-36.

[2] Jisun An, Daniele Quercia, and Jon Crowcroft. 2013. Fragmented social me dia: a look into selective exposure to political news. In Proceedings of the 22nd International Conference on World Wide Web. ACM, 51-52.

[3] Anja Bechmann and Kristoffer L. Nielbo. 2018. Are We Exposed to the Same "News" in the News Feed? Digital Journalism (09 2018), 1-13. https://doi.org/10 1080/21670811.2018.1510741

[4] Shlomo Benartzi, Ehud Peleg, and Richard H Thaler. 2013. Choice architecture and retirement saving plans. The behavioral foundations of public policy (2013), 245-263.

[5] Sushil Bikhchandani, David Hirshleifer, and Ivo Welch. 1992. A theory of fads, fashion, custom, and cultural change as informational cascades. Fournal of political Economy 100, 5 (1992), 992-1026.

[6] Jesús Bobadilla, Fernando Ortega, Antonio Hernando, and Abraham Gutiérrez. 2013. Recommender systems survey. Knowledge-based systems 46 (2013), 109132 .

[7] Federica Cena, Amon Rapp, Cataldo Musto, and Pasquale Lops. 2018. Towards a Conceptual Model for Holistic Recommendations. In Adjunct Publication of the 26th Conference on User Modeling, Adaptation and Personalization (UMAP '18). ACM, New York, NY, USA, 207-210. https://doi.org/10.1145/3213586.3225248

[8] Paul Davidsson. 2002. Agent based social simulation: A computer science view. Journal of artificial societies and social simulation 5, 1 (2002).

[9] Michela Del Vicario, Fabiana Zollo, Guido Caldarelli, Antonio Scala, and Walter Quattrociocchi. 2017. Mapping social dynamics on Facebook: The Brexit debate Social Networks 50 (2017), 6-16.

[10] Farzad Eskandanian, Bamshad Mobasher, and Robin Burke. 2017. A Clustering Approach for Personalizing Diversity in Collaborative Recommender Systems. In Proceedings of the 25th Conference on User Modeling, Adaptation and Personalization (UMAP '17). ACM, New York, NY, USA, 280-284. https //doi.org/10.1145/3079628.3079699

[11] Soodeh Farokhi, Pooyan Jamshidi, Ivona Brandic, and Erik Elmroth. 2015. Selfadaptation challenges for cloud-based applications: A control theoretic perspec tive. In 10th International Workshop on Feedback Computing, Vol. 2015

[12] Emilio Ferrara. 2017. Disinformation and social bot operations in the run up to the 2017 French presidential election. First Monday 22, 8 (2017). http:// firstmonday.org/ojs/index.php/fm/article/view/8005

[13] Emilio Ferrara, Onur Varol, Clayton Davis, Filippo Menczer, and Alessandro Flammini. 2016. The rise of social bots. Commun. ACM 59, 7 (2016), 96-104.

[14] Corrado Gini. 1921. Measurement of inequality of incomes. The Economic fournal 31, 121 (1921), 124-126.

[15] Jacob Goldenberg, Barak Libai, and Eitan Muller. 2001. Talk of the network: A complex systems look at the underlying process of word-of-mouth. Marketing letters 12, 3 (2001), 211-223.

[16] Mario Haim, Andreas Graefe, and Hans-Bernd Brosius. 2018. Burst of the Filter Bubble? Digital fournalism 6, 3 (2018), 330-343. https://doi.org/10.1080/21670811. 2017.1338145 arXiv:https://doi.org/10.1080/21670811.2017.1338145

[17] Eduardo Hargreaves, Claudio Agosti, Daniel Menasché, Giovanni Neglia, Alexandre Reiffers-Masson, and Eitan Altman. 2018. Biases in the Facebook News Feed: a Case Study on the Italian Elections. arXiv preprint arXiv:1807.08346 (2018).

[18] Kathleen Hall Jamieson and Joseph N Cappella. 2008. Echo chamber: Rush Limbaugh and the conservative media establishment. Oxford University Press.

[19] Daniel Kahneman. 2003. Maps of bounded rationality: Psychology for behavioral economics. American economic review 93, 5 (2003), 1449-1475

[20] David Kempe, Jon Kleinberg, and Éva Tardos. 2003. Maximizing the spread of influence through a social network. In Proceedings of the ninth ACM SIGKDD international conference on Knowledge discovery and data mining. ACM, 137-146.

[21] Joseph A Konstan and John Riedl. 2012. Recommender systems: from algorithms to user experience. User modeling and user-adapted interaction 22, 1-2 (2012), 101-123.

[22] Shyong K Lam and John Riedl. 2004. Shilling recommender systems for fun and profit. In Proceedings of the 13th international conference on World Wide Web. ACM, 393-402.

[23] David MJ Lazer, Matthew A Baum, Yochai Benkler, Adam J Berinsky, Kelly M Greenhill, Filippo Menczer, Miriam J Metzger, Brendan Nyhan, Gordon Pennycook, David Rothschild, et al. 2018. The science of fake news. Science 359, 6380 (2018), 1094-1096.

[24] Marc Lelarge. 2012. Diffusion and cascading behavior in random networks. Games and Economic Behavior 75, 2 (2012), 752-775.

[25] Jure Leskovec, Ajit Singh, and Jon Kleinberg. 2006. Patterns of influence in a recommendation network. In Pacific-Asia Conference on Knowledge Discovery and Data Mining. Springer, 380-389.

[26] Marco Lippi and Paolo Torroni. 2015. Argument mining: A machine learning perspective. In International Workshop on Theorie and Applications of Formal Argumentation. Springer, 163-176.
[27] Bing Liu and Lei Zhang. 2012. A survey of opinion mining and sentiment analysis. In Mining text data. Springer, 415-463.

[28] Andrew L Maas, Raymond E Daly, Peter T Pham, Dan Huang, Andrew Y Ng, and Christopher Potts. 2011. Learning word vectors for sentiment analysis. In Proceedings of the 49th annual meeting of the association for computational linguistics: Human language technologies-volume 1. Association for Computational Linguistics, 142-150.

[29] Bamshad Mobasher, Robin Burke, Runa Bhaumik, and Chad Williams. 2007. Toward trustworthy recommender systems: An analysis of attack models and algorithm robustness. ACM Transactions on Internet Technology (TOIT) 7, 4 (2007), 23.

[30] Judith Möller, Damian Trilling, Natali Helberger, and Bram van Es. 2018. Do not blame it on the algorithm: An empirical assessment of multiple recommender systems and their impact on content diversity. Information, Communication \& Society 21, 7 (2018), 959-977.

[31] Andrea Montanari and Amin Saberi. 2010. The spread of innovations in social networks. Proceedings of the National Academy of Sciences 107, 47 (2010), 2019620201.

[32] Fred Morstatter, Yunqiu Shao, Aram Galstyan, and Shanika Karunasekera. 2018. From Alt-Right to Alt-Rechts: Twitter Analysis of the 2017 German Federal Election. In Companion of the The Web Conference 2018 on The Web Conference 2018. International World Wide Web Conferences Steering Committee, 621-628.

[33] Friedrich Wilhelm Nietzsche. 1913. The Complete Works of Friedrich Nietzsche: Human, all-too-human. Vol. 7. TN Foulis.

[34] Kieron O'Hara and David Stevens. 2015. Echo chambers and online radicalism: Assessing the Internet's complicity in violent extremism. Policy \& Internet 7, 4 (2015), 401-422.

[35] Eli Pariser. 2011. The filter bubble: What the Internet is hiding from you. Penguin UK.

[36] Norberto Patrignani, Diane Whitehouse, and Monica Gemo. 2017. Forget About Privacy... or Not?. In IFIP International Summer School on Privacy and Identity Management. Springer, 76-85.

[37] Nathaniel Persily. 2017. The 2016 US Election: Can democracy survive the internet? Journal of democracy 28, 2 (2017), 63-76.

[38] Thomas Piketty. 2015. About capital in the twenty-first century. American Economic Review 105, 5 (2015), 48-53.

[39] Daniel M Romero, Brendan Meeder, and Jon Kleinberg. 2011. Differences in the mechanics of information diffusion across topics: idioms, political hashtags, and complex contagion on twitter. In Proceedings of the 20th international conference on World wide web. ACM, 695-704.

[40] LJ Russell. 1938. A Critical Exposition of the Philosophy of Leibniz. By Russell Bertrand, New impression with a new preface (London: George Allen \& Unwin, Ltd. 1937. Pp. xxiii+ 311. Price 12s. 6d.). Philosophy 13, 50 (1938), 217-220.

[41] Sandip Sen, Chad Crawford, Zenefa Rahaman, and Yücel Osman. 2018. Agents for Social (Media) Change. In Proceedings of the 17th International Conference on Autonomous Agents and Multiagent Systems (AAMAS 2018). Stockholm.

[42] Chengcheng Shao, Giovanni Luca Ciampaglia, Onur Varol, Alessandro Flammini, and Filippo Menczer. 2017. The spread of fake news by social bots. arXiv preprint arXiv:1707.07592 (2017), 96-104.

[43] Elisa Shearer and Jeffrey Gottfried. 2017. News use across social media platforms 2017. Pew Research Center, fournalism and Media (2017).

[44] N Craig Smith, Daniel G Goldstein, and Eric J Johnson. 2013. Choice without awareness: Ethical and policy implications of defaults. Fournal of Public Policy \& Marketing 32, 2 (2013), 159-172.

[45] Cass R. Sunstein. 2001. Republic.Com. Princeton University Press, Princeton, NJ, USA.

[46] Richard H Thaler and Cass R Sunstein. 2003. Libertarian paternalism. American economic review 93, 2 (2003), 175-179.

[47] Onur Varol, Emilio Ferrara, Clayton Davis, Filippo Menczer, and Alessandro Flammini. 2017. Online Human-Bot Interactions: Detection, Estimation, and Characterization. https://aaai.org/ocs/index.php/ICWSM/ICWSM17/paper/ view/15587/14817

[48] Duncan J Watts. 2002. A simple model of global cascades on random networks. Proceedings of the National Academy of Sciences 99, 9 (2002), 5766-5771.

[49] Michael Wooldridge. 2009. An introduction to multiagent systems. John Wiley \& Sons.

[50] Yafit Lev-Aretz. 2018. Facebook and the perils of a personalized choice architecture. http://social.techcrunch.com/2018/04/24/ facebook-and-the-perils-of-a-personalized-choice-architecture/ 